\title{
A toe that pointed the wrong way: An unusual presentation of tetanus
}

\author{
Krishnarpan Chatterjee, Anirban Ghosh, Rimi Som Sengupta \\ Department of Medicine, ESI-PGIMSR and ESI Medical College, Joka, Kolkata, West Bengal, India
}

\section{ABSTRACT}

We report the case of a 15-year-old girl who was initially diagnosed to have a striatal toe. Her condition progressed and she later developed clinical features consistent with tetanus. History of blunt trauma to nose was elicited retrospectively. Antimicrobial therapy with metronidazole and both active and passive immunization was started immediately. The patient went on to make a complete recovery.

Key words: Striatal toe, tetanus, striatal toe, tetanus, post-exposure prophylaxis

\section{Introduction}

Tetanus is a disease characterized by hypertonia and muscular spasms due to the toxin tetanospasmin formed by Clostridium tetani. Tetanus continues to be widespread, especially in the developing world though incidences have come down due to immunization. Localized tetanus is a rare variant affecting muscles near the site of inoculation. It is usually mild but may progress to generalized tetanus. We present a case of tetanus in a young girl which initially mimicked a striatal toe though the potential cause of inoculation was trauma to nose. This rare presentation led to diagnostic confusion. The diagnosis was made when she developed generalized tetanus. Prompt institution of treatment led to a complete recovery.

\section{Case Report}

A 16-year-old girl presented with a history of sudden onset of pain in left leg with difficulty in walking for 2 days. The pain was intermittent and affecting her sleep.

\begin{tabular}{|l|l|}
\hline \multicolumn{2}{|c|}{ Access this article online } \\
\hline Quick Response Code: & Website: \\
\hline & www.ruralneuropractice.com \\
\hline & \\
\hline
\end{tabular}

It was associated with intermittent abnormal upturned posture of the big toe. There was no history of swelling of leg. There was no history of any other abnormal movements. There was no history of similar complaints in the past. She had no history of any drug intake. On examination, her general physical examination was normal. Gait was antalgic. Higher mental function and cranial nerves were normal. Tone was increased below the knee on the left side. An upturned big toe without fanning of other toes was seen on the left [Figure 1]. This abnormal posture was sustained and increased on the sensory stimulus. Ankle jerk could not be elicited on the left side. A clinical diagnosis of a striatal toe was made and the patient was admitted for further evaluation [Figures 1 and 2]. The abnormal posture of the foot was maintained during sleep. Contrast-enhanced magnetic resonance imaging of the brain revealed no abnormality of the basal ganglia. Liver function test was normal and slit lamp examination did not show a $\mathrm{KF}$ ring. Corrected serum calcium was $9.8 \mathrm{mg} / \mathrm{dL}$. There was no response to benzodiazepines. On the $3^{\text {rd }}$ day after admission, she developed stiffness of bilateral lower limbs followed by episodic spasms of the back and neck. A clinical diagnosis of tetanus was made. There were no signs of autonomic hyperactivity and she did not have any seizures. On further probing, patient's mother revealed that she had suffered a fall a day before onset of her symptoms and hurt her nose. She had received tetanus toxoid (TT) $0.5 \mathrm{ml}$ intramuscularly afterward. Her mother said that she had received her primary series of TT in infancy and received her last booster 6 years

Address for correspondence:

Dr. Krishnarpan Chatterjee, B-7/11, Diamond Park, Diamond Hoarbour Road, Joka, Kolkata - 700 104, West Bengal, India.

E-mail: krishnarpan.chatterjee@gmail.com 


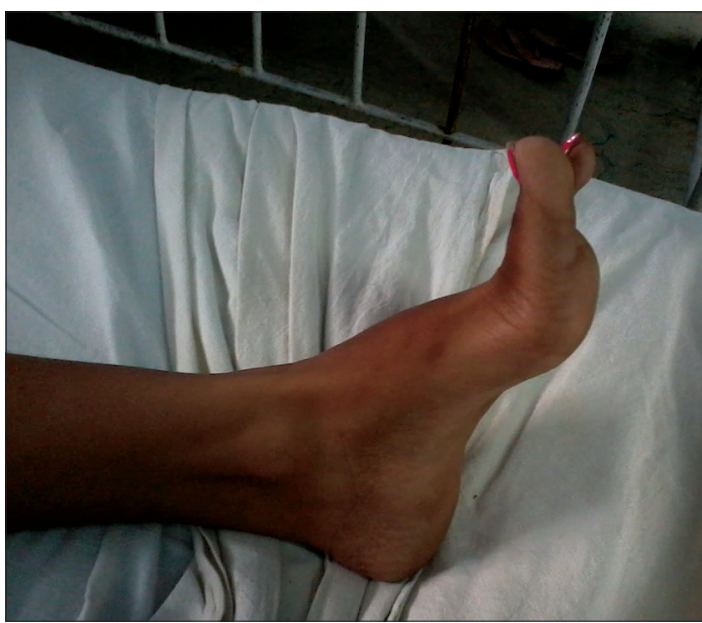

Figure 1: Striatal toe

back at around 10 years of age. She was given tetanus immunoglobulin (TIG) 5000 units intramuscularly stat and also started on intravenous metronidazole and diazepam infusion. A full course of TT vaccination was also started. On the $5^{\text {th }}$ day after admission, she developed mild trismus and opisthotonus completing the clinical picture of generalized tetanus. She did not develop any cranial nerve palsies or respiratory difficulty. She started showing signs of recovery by day 10 and made a complete recovery in 3 weeks from onset of symptoms.

\section{Discussion}

Tetanus is caused by the toxin tetanospasmin released by the germinating spores of the anaerobic gram-positive bacilli $C$. tetani. As spores of the bacilli occur in the soil, tetanus frequently occurs following a contaminated wound, but cases have also been reported after dental procedures, surgeries, burns, intravenous drug abuse, and intramuscular injections. ${ }^{[1,2]}$

Tetanus has been clinically classified as generalized, localized, cephalic, and neonatal. ${ }^{[2]}$ Our patient presented with a localized form of tetanus affecting initially the left leg that mimicked a striatal toe even though there was no local injury in the leg. The potential site of inoculation being in the head and neck region was unusual. It is possible that our patient had another insignificant trauma to her leg that had gone unnoticed. Tetanus has also been reported without a clear history of inoculation. ${ }^{[3]}$

A striatal toe is a finding in clinical neurology that reliably localized the lesion to the caudate nucleus and putamen. The absence of fanning of the rest of the toes differentiates the upturned big toe from the Babinski

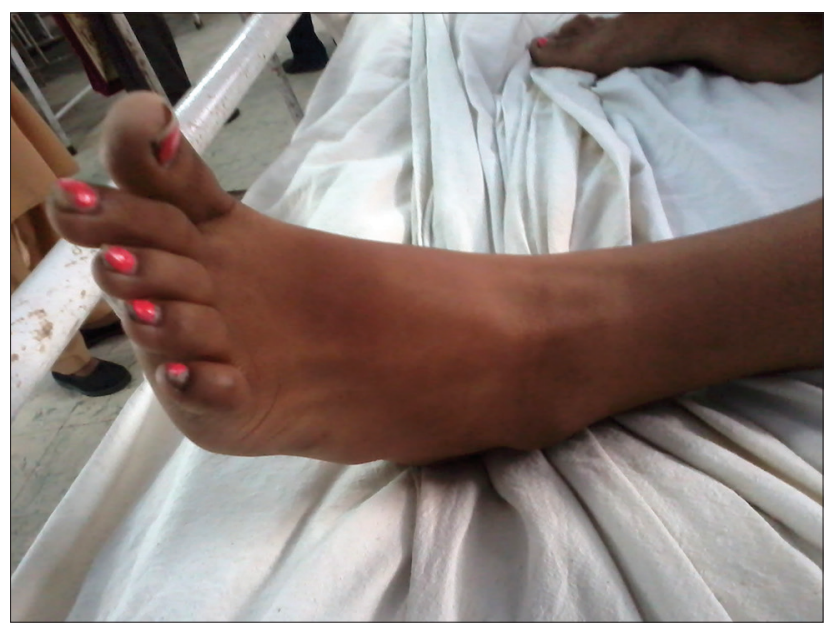

Figure 2: Extension of great toe with flexion of other toes

extensor plantar response. Tetanus presenting initially with the classic clinical picture of striatal toe has not been reported before. Thus, it is important to keep localized tetanus in the differential diagnosis of extrapyramidal disorders presenting with such spasticity, especially in the absence of definite history of trauma. Dystonias can be ruled out in such cases by checking for resolution after a dose of benztropine.

Severity of tetanus can be graded according to the Ablett classification into four grades..$^{[2]}$ According to the Ablett classification, our patient had grade one severity and thus a better prognosis.

The World Health Organization recommends a 5 dose schedule for tetanus vaccination while in India additional doses are recommended in the Universal Immunization Program (UIP). ${ }^{[4,5]}$ Our patient had received three doses as DTP in infancy and another dose between 12 and 24 months of age. She also had received two booster doses of DTP and Td at 5 years and 10 years according to her mother as per the UIP.

Postexposure prophylaxis can prevent the development of tetanus. The advisory committee on immunization practices of the centers for disease control does not recommend either Td vaccination or TIG for patients who have received more than three doses of TT with the last dose within 5 years. ${ }^{[6]} \mathrm{Td}$ is recommended in clean wounds with last booster $>10$ years back and other wounds with last booster $>5$ years back. TIG should be given in unclean wounds when vaccination status is unknown or patient has received $<3$ doses. Tetanus prone wounds are $>6 \mathrm{~h}$ old, $>1 \mathrm{~cm}$ deep, stellate, ischemic, denervated, contaminated or infected. ${ }^{[7]}$ However minor wounds can also lead to tetanus. ${ }^{[8]}$ postexposure passive immunization involves $250 \mathrm{U}$ of TIG given 
intramuscularly. Some of the TIG may be injected into the wound if it is identifiable. Meticulous wound care is also important. Our patient had received TT immunization at the time of primary wound care as per the recommendations. She did not receive TIG as she had received more than 3 doses of immunization in the past. Identification of vulnerable patients by testing for protective tetanus antibodies is not viable in our setting. Our patient developed tetanus in spite of having received TT postexposure. This may be due to a defect in the patients humoral immunity due to which she couldn't mount an antibody response. Rapid bedside tests to screen for tetanus immunization status have been found to be more effective than medical interview and wound assessment in some studies, but cost effectiveness remains an issue. ${ }^{[9,10]}$

Wound care, antimicrobial therapy with metronidazole and human TIG are cornerstones of therapy of established tetanus. Metronidazole has been shown to be a better alternative to penicillin. ${ }^{[11]}$ TIG is given in a dose of 3000-6000 units intramuscularly. ${ }^{[12]}$ A full course of active immunization with TT should be started early as neither clinical infection nor TIG provide long-term immunity. Muscular spasms may be controlled by intravenous benzodiazepines. Respiratory distress requires intubation and mechanical ventilation. ${ }^{[2]}$

Cases of tetanus continue to come to medical attention in spite of widespread immunization in this age and time. We must be alert to the possibility and institute prompt treatment after a clinical diagnosis as early passive immunization reduces morbidity and mortality.

\section{References}

1. Burgess JA, Wambaugh GW, Koczarski MJ. Report of case: Reviewing cephalic tetanus. J Am Dent Assoc 1992;123:67-70.

2. Cook TM, Protheroe RT, Handel JM. Tetanus: A review of the literature. Br J Anaesth 2001;87:477-87.

3. Ihara F, Otsuka Y. Experience of two cases of tetanus without a clear history of trauma. Nihon Jibiinkoka Gakkai Kaiho 2014;117:41-5.

4. World Health Organization. Tetanus vaccine: WHO position paper. Wkly Epidemiol Rec 2006;81:198-207.

5. Government of India. Immunization Handbook for Medical Officers. New Delhi: Ministry of Health and Family Welfare, Nirman Bhawan, Government of India; 2009. p. 21.

6. Kretsinger K, Broder KR, Cortese MM, Joyce MP, Ortega-Sanchez I, Lee GM, et al. Preventing tetanus, diphtheria, and pertussis among adults: Use of tetanus toxoid, reduced diphtheria toxoid and acellular pertussis vaccine recommendations of the Advisory Committee on Immunization Practices (ACIP) and recommendation of ACIP, supported by the Healthcare Infection Control Practices Advisory Committee (HICPAC), for use of Tdap among health-care personnel. MMWR Recomm Rep 2006;55:1-37.

7. Hsu SS, Groleau G. Tetanus in the emergency department: A current review. J Emerg Med 2001;20:357-65.

8. Rhee P, Nunley MK, Demetriades D, Velmahos G, Doucet JJ. Tetanus and trauma: A review and recommendations. J Trauma 2005;58:1082-8.

9. Colombet I, Saguez C, Sanson-Le Pors MJ, Coudert B, Chatellier G, Espinoza $\mathrm{P}$, et al. Diagnosis of tetanus immunization status: Multicenter assessment of a rapid biological test. Clin Diagn Lab Immunol 2005;12:1057-62.

10. Hatamabadi HR, Abdalvand A, Safari S, Kariman H, Dolatabadi AA, Shahrami A, et al. Tetanus Quick Stick as an applicable and cost-effective test in assessment of immunity status. Am J Emerg Med 2011;29:717-20.

11. Ahmadsyah I, Salim A. Treatment of tetanus: An open study to compare the efficacy of procaine penicillin and metronidazole. $\mathrm{Br} \mathrm{Med}$ J (Clin Res Ed) 1985;291:648-50.

12. Blake PA, Feldman RA, Buchanan TM, Brooks GF, Bennett JV. Serologic therapy of tetanus in the United States, 1965-1971. JAMA 1976;235:42-4.

How to cite this article: Chatterjee K, Ghosh A, Sengupta RS. A toe that pointed the wrong way: An unusual presentation of tetanus. J Neurosci Rural Pract 2016;7:150-2.

Source of Support: Nil. Conflict of Interest: None declared. 